Mumerical Treatment of the Axial Singularity

In a Flux-Coordinate System

\title{
Gloletta Kuo-Petravic
}

Plasma Physics Laboratory, Princeton University

P.0. Box 451

Princeton, New Jersey 08544

In the realistic simulation of physical systems whether in 2-D or 3-D there arise often situations where the fluid flow has a preferred direction. An example of this is a magnetically confined plasma where the flow is predominently along the magnetic fleld lines. To avoid a large unerical diffusion and hence inaccuracy, it is often necessary to adopt a flux coorfinate systed with coordinates following slosely the rontours given naturally by the physics of the problem, an example for this is shown in fig. 1. In this system radial motion is measured by the flux function $\psi=\oint \vec{B} \cdot \vec{d} \vec{A}$, where $\vec{B}$ is $c$ he magnetic fleld and $\vec{A}$ an enclosed area whose boundary is traced out by the intersections of a magnetic field line with a poloital plane. The angular variable $\theta_{0}$ is measured from the axis which is at the center of the set of nested $\psi$ surfaces. Th1s point at $\psi=0$ corresponds to multivalues of $\theta_{0}$ and this causes most general numerical methods to break down. However we shall show here that 1 t is prosible and efficient in computer time is overlay the $\psi, \theta_{0}$ mesh with a rectangular $x, y$ mesh near the origin. Wth this transformation the pecullarities of the singular point disappea: and ordinary numerical techniques can be carried through

We shall use as an example a problem we have encountered in our 
simulation of a plasma in an asymmetric torus $[1,2]$. Here the fact that a flux surface is also an equipotential surface glves additional incentive for the use of flux coordinates. The equations for the drift motion of lons in a magnetic fleld can be cast in a very slople Hamiltonian form which can be easily integrated [2] If the flux coordinates $e_{0}, x$ are used. re $\psi$ is the toroidal flux, $\theta_{0}$ a polofdal argle measured from the magnetic axt and $\chi$ is a coordinate along the magnetic field line. Since $x$ does not ent Intw our essentlally 2 dimensional problem we shall henceforth restrlct ourselves co $\theta_{0}, \psi$ only.

Fe drift equations are:

$$
\begin{aligned}
& \frac{d \theta_{0}}{d t}=-A \frac{\partial B}{\partial \psi}-\frac{\partial \Phi}{\partial \psi} \\
& \frac{d \psi}{d t}=A \frac{\partial B}{\partial \theta_{0}}
\end{aligned}
$$

where $B$ is the magnetic field and

$$
\begin{aligned}
& A=\mu+\rho_{\|}^{2} B \text {, where } \mu=\text { the magnetic moment, } \\
& \rho_{n}=\text { the parallel Lamor radius, and } \\
& \Phi(\psi)=\text { electric potential. }
\end{aligned}
$$

When these equations are integrated very close to $\psi=0$, the singularity at the axis manifests itself most commonly as the unphysical condition $\psi<0$ when an Integration scheme like the 4th order Range-Kutta is used. Although the probability of this occuring is in general of the order of the ratio of a few times $\pi \nabla \psi^{2}$ to the total flux area, where $\nabla \psi$ is the change in $\psi$ in one time step, (in our case this ratio is around $10^{-4}$ ), it is very inconveniert 
espectally when particles have long confinement times and the runs abort before data collection is complete.

In our method we transform to an $x, y$ system using circularized flix surfaces :

$$
\begin{aligned}
& x=(2 \psi)^{1 / 2} \cos \theta_{0} \\
& y=(2 \psi)^{1 / 2} \sin \theta_{0} .
\end{aligned}
$$

We then advance the $x, y$ coordinates in time instead of the $\theta_{0}, \psi$ coordinates using the equations:

$$
\begin{aligned}
& \frac{d x}{d t}=\frac{1}{(2 \psi)^{1 / 2}} \cos \theta_{0}\left(\frac{d \psi}{d t}\right)-(2 \psi)^{1 / 2} \sin \theta_{0}\left(\frac{d \theta_{0}}{d t}\right) \\
& \frac{d y}{d t}=\frac{1}{(2 \psi)^{1 / 2}} \sin \theta_{0}\left(\frac{d \psi}{d t}\right)+(2 \psi)^{1 / 2} \cos \theta_{0}\left(\frac{d \theta_{0}}{d t}\right)
\end{aligned}
$$

Since for any given $x, y$ the corresponding $\theta_{0}, \Psi$ can be calculated everywhere using the equations (II), we are able to derive the values of $d \psi / d t$ and $\mathrm{d} \theta_{0} / \mathrm{d}$ from equations (I) once the gradients of $B$ in the $\theta_{0}, \psi$ coordinate system are known. Therefore:

$$
\begin{aligned}
& \frac{\mathrm{dx}}{\mathrm{dt}}=\frac{A \cos \partial_{0}}{(2 \psi)^{1 / 2}} \frac{\partial B}{\partial \theta_{0}}+(2 \psi)^{1 / 2} \sin \theta_{0}\left[\frac{\partial \Phi}{\partial \psi}+A \frac{\partial B}{\partial \psi}\right] \\
& \frac{\mathrm{dy}}{d t}=\frac{A \sin \theta_{0}}{(2 \psi)^{1 / 2}} \frac{\partial B}{\partial \theta_{0}}+(2 \psi)^{1 / 2} \cos \theta_{0}\left[\frac{\partial \Phi}{\partial \psi}+A \frac{\partial B}{\partial \psi}\right]
\end{aligned}
$$

The magnetic ficld near the axis can be expanded in the form: 


$$
\mathrm{B}=\sum_{\mathrm{n}, \mathrm{m}} \psi^{|\mathrm{w}| / 2}\left(\mathrm{a}+\mathrm{b} \psi+\mathrm{c} \psi^{2} \ldots\right) \cos \left(\omega \chi-\mathrm{m} \theta_{0}\right)
$$

$$
\begin{aligned}
& \text { where } n=\text { toroldal more number } \\
& \omega=\text { pololdal mode number } \\
& \omega=(n-m l) / g \\
& g=\text { total polsidal current } \\
& l=\text { rotational transform }
\end{aligned}
$$

from which

$$
\begin{aligned}
& \frac{\partial B}{\partial \theta}=\sum_{0, m} \psi|m| / 2\left(a+b \psi+c \psi^{2}+d \psi^{3}\right) s 1 n\left(\omega x-m \theta_{0}\right) \\
& \frac{\partial B}{\partial \psi}=\sum_{n, m}\left[\frac{|m|}{2} \psi\left(\frac{|m|}{2}-1\right)\left(a+b \psi+c \psi^{2}+d \psi^{3}\right) \cos \left(\omega \chi-m_{0}\right)\right. \\
& +\psi^{|m| / 2}\left(b+2 c \psi+3 d \psi^{2}\right) \cos \left(\omega \chi-m \theta_{0}\right) \\
& \left.-\psi^{|m| / 2}\left(a+b \psi+c \psi^{2}+d \psi^{3}\right) \sin \left(\omega \chi-m \theta{ }_{0}\right)\left[\frac{m l^{\prime}}{g} \chi\right)\right]
\end{aligned}
$$

For $|m|=$ I the first term of the second equation in (VI) tends to infinity as $\psi$ goes to 0 . This 1 s of course the reason why eqns (I) are ot useful near the orfgin. Gowever when (VI) is Incoporated into the Cartesian formulation In (IV) the multiplying factor $\psi^{1 / 2}$ removes the singularity in the first term of $\frac{\partial B}{\partial \psi}$. This must hold for any $B$ which is physlcal as Eq. (IV) is ralld so long as $B$ is analytic near $\psi=0$.

We have Inplemented the algorithm outlined above and FIg. (2) shows the projected trajectory in $\theta_{0}, \psi$ plane of a particle whose path straddles the boundary at $\psi=\psi_{c}$. For $\psi<\psi_{c}$ the particle position is advanced in $x, y$ space while for the region $\psi_{c}<\psi<\psi_{a}$ the computation is entirely in $\theta_{0}$, $\psi$ space. Here $\psi_{c}=10^{-4}$ and $\psi_{a}=1$. The cholce of $\psi_{c}$ denends mostly on the maxtmum $\Delta \psi$ the particle makes per time sté in the reglon around $\psi=0$. To prevent the 
particle from crossing tine axial region in one step starting from $\psi_{>} \psi_{c}$, it Is aesirable to make $\psi_{c}^{\prime} \Delta \psi \geqslant 10$

Major alteration to the computer program 1 s only required in the subroutine slpplying the RHS of (IV) to the Runge-Kutta control subroutine. In order to retaln as much as possible the vectorization benefits of the CRAYI complier, we make the same array store elther $\left(\theta_{0}, \psi\right)$ or $(x, y)$ depending on the value 0 or 1 of a switch. This switch is used to direct the flow to elther the $\left(\theta_{0}, \psi\right)$ or the $(x, y)$ blocks of code. Testing on $\psi$ to find out if the particle has just entered or left the $x, y$ computational region given by $\psi<\psi_{c}$ is performed once per time step in the main program. A alternative solution when eqns (IV) are only used when $\Psi_{c} \ll I$ is to drop all higher terms in the polynomial for B, (V), keeping only the constant term. This was found to reduce the computer time required for one timestep by $20 \%$.

Acknowl edgment

This work was supported by Unted States Department of Energy Contract No. $\mathrm{DE}-\mathrm{ACO} 2-76-\mathrm{CHO} 3073$.

I wish to thank R.C. Grimm and R. B. White for their helpful discussions. 


\section{References}

[1] G. Kuo-Petravic, A. H. Boozer, J. Rome, and R. Fowler to appear in J. Compt . Phys . (1982).

[2] A. H. Boozer and G. Kuo-Petrav1c, Phys. Fulds 24, 831 (1981).

[3] A. H. Boozer, Phys. Fluids (1982). 
Figure Captions

Fig. 1. Schematic diagram of a 2-D flux coordinate system $\theta_{G}, \psi$.

Fig. 2. Prajections of a trajectory of a particle in an asymetric toroidal plasma device. Points inside the circle $\psi=\psi_{c}$ are calculated in $(x, y)$ system and points outside in $\left(\theta_{0}, 4\right)$ system. 
\# 82 T 0114

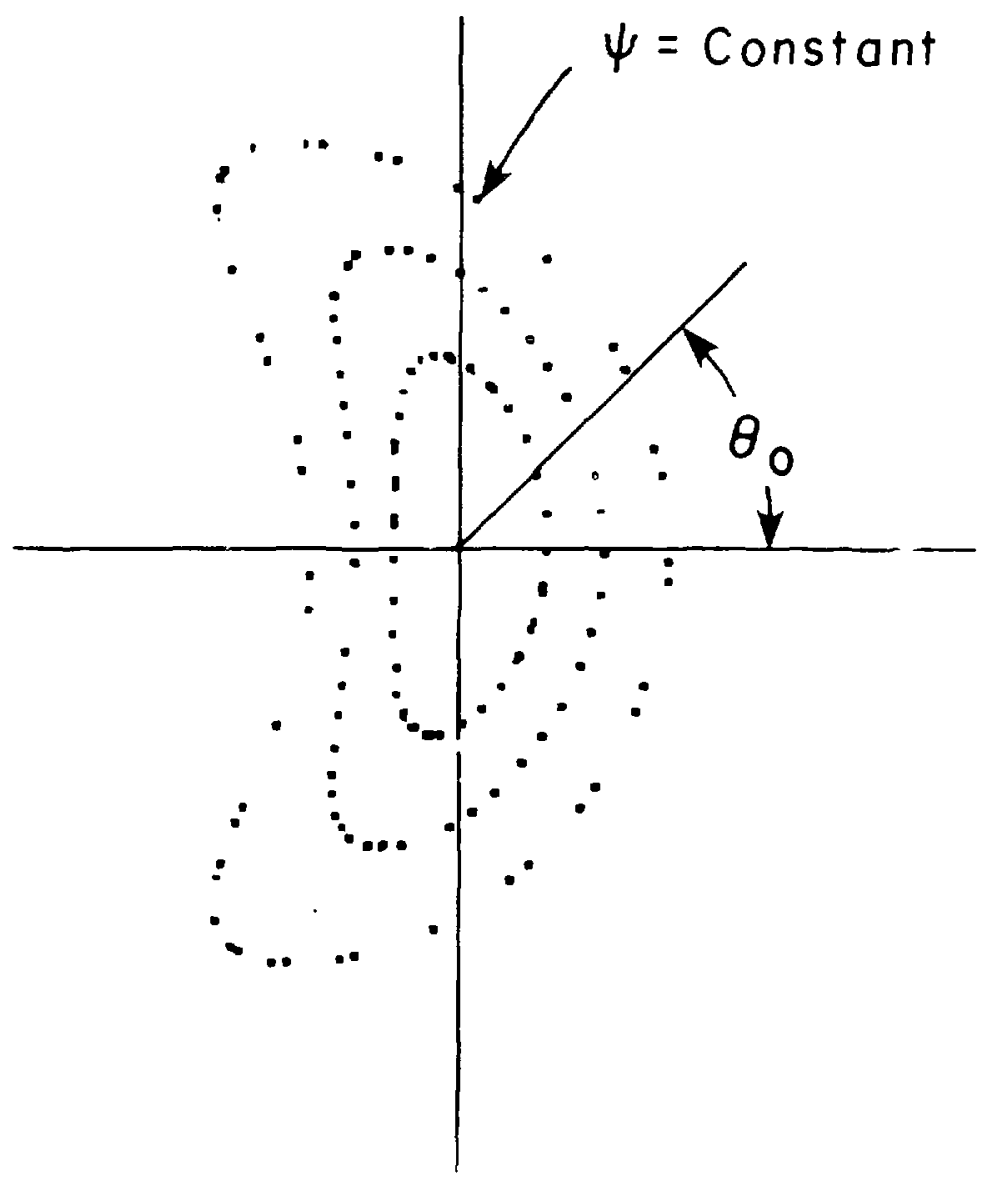

Fig. 1 
9

\# 82 T 0113

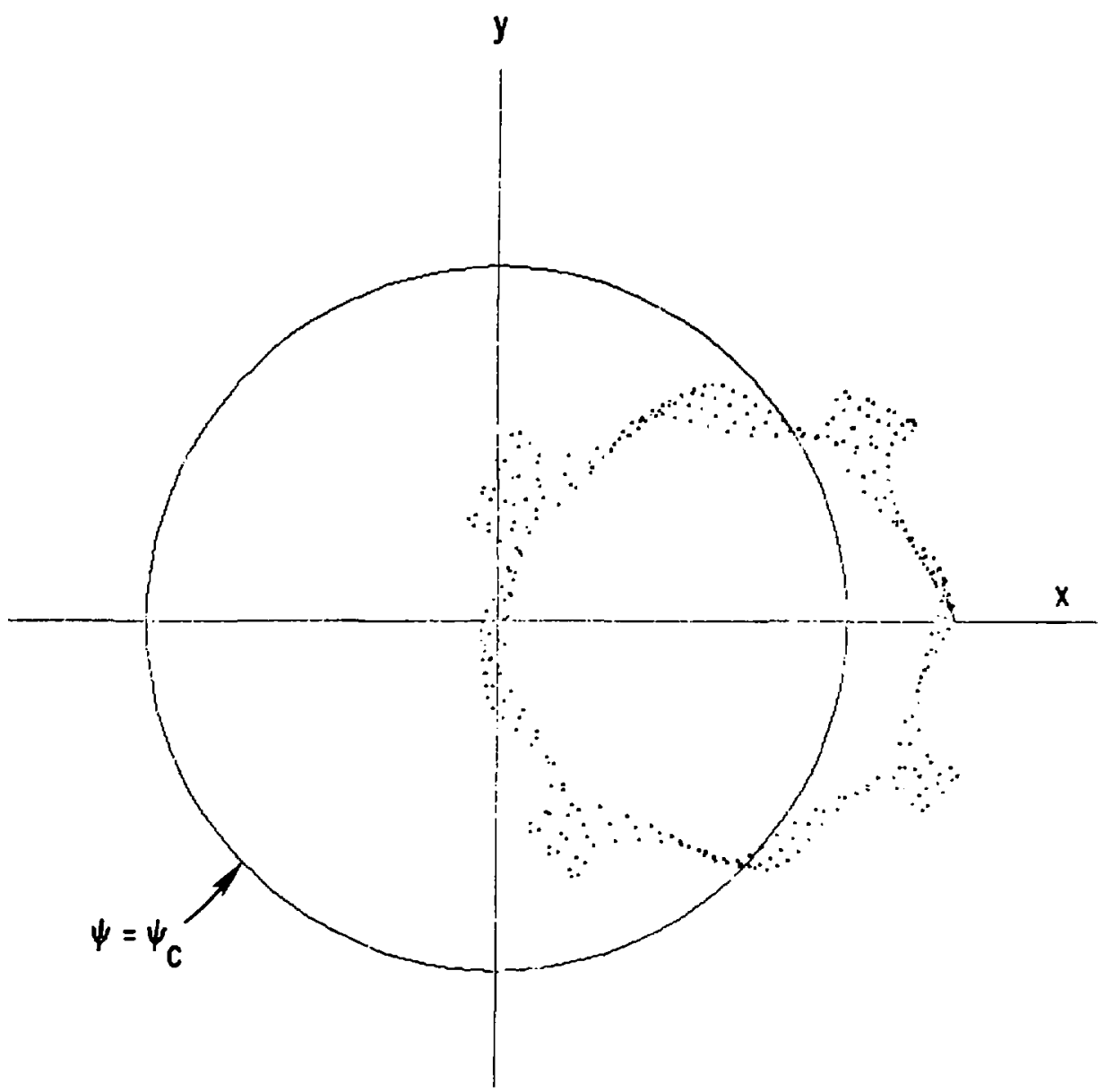

Fig. 2 